\title{
Source-Side Injection Modeling by Means of the Spherical-Harmonics Expansion of the BTE
}

\author{
M. Lorenzini, D. Wellekens, L. Haspeslagh and J. Van Houdt \\ IMEC_-Interuniversity Microelectronics Center \\ Kapeldreef 75, 3001 Leuven, Belgium \\ Martino.Lorenzini@imec.be
}

\begin{abstract}
The source-side injection current in a split-gate structure has been modeled starting from the electron energy distribution along the $\mathrm{Si}_{-} \mathrm{SiO}_{2}$ interface provided by the deterministic solution of the Boltzmann Transport Equation in the semiconductor domain. When accounting for parallel momentum conservation, the order of magnitude of the current and its dependence on applied voltages is successfully reproduced.
\end{abstract}

\section{Introduction}

Standard simulation tools, based on transport models derived from the moments of the Boltzmann Transport Equation (BTE), are commonly used in technology development. However, being approaches that employ macroscopic averages, they do not fully capture non-local effects, such as carrier heating, thus making the modeling of gate currents rather questionable. On the other hand, Monte Carlo (MC) calculations are still characterized by a considerable computational burden, which excludes them from extensive design studies. In recent years, the spherical-harmonics expansion (SHE) of the BTE [1] has proven to be a valuable tool for the analysis of semiconductor devices. The main advantage of the method is the possibility to compute the energy-distribution function of the carriers in a deterministic way, which is computationally many times faster than MC simulators. In this work, the BTE solver HARM [2] is used to calculate the electron energy distribution along the silicon surface in a HIMOS ${ }^{\mathrm{TM}}$ memory device [3], programmed by source-side injection. Such a distribution is then post-processed to evaluate the floating-gate current.

\section{Device Description and Experimental Data}

The HIMOS ${ }^{\mathrm{TM}}$ concept uses a split-gate transistor, consisting of a floating-gate channel in series with a control-gate channel (Fig. 1). A third electrode, located on the field region, couples a relatively high voltage onto the floating gate, so that, during programming, the floating-gate potential is always higher than the potentials of both drain and control gate. Samples used in this study have been manufactured in a $0.18 \mu \mathrm{m}$ technology and feature a $0.21 \mu \mathrm{m}$-long control gate and a $0.42 \mu \mathrm{m}$-long floating gate. A $7.5 \mathrm{~nm}$ oxide isolates the gates from the silicon substrate. Floating-gate currents have been determined from the programming characteristics, so that the total displaced charge is very small and the influence of trapping is ruled out [3]. Thus, $I_{f g}=C_{p} d V_{t h} / d t$, where $C_{p}$ is the coupling capacitance between floating-gate and program-gate and $V_{t h}$ 


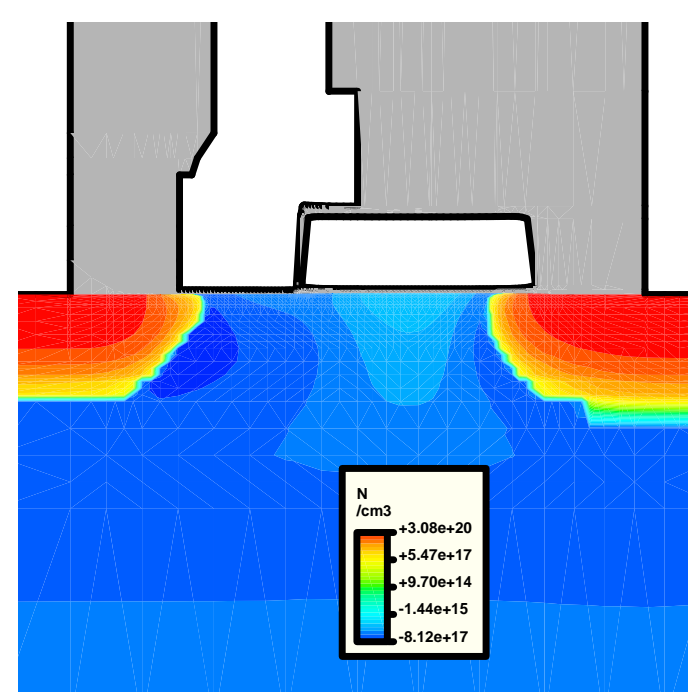

Figure 1: Simulated split-gate structure and doping distribution.

the measured threshold voltage. The floating-gate potential is derived from a capacitor model of the cell, once the relevant coupling coefficients have been measured [3].

\section{Physical Models}

HARM solves the following system of equations, which results from the SHE of the stationary BTE after truncation of the expansion to first order [1]:

$-\nabla_{r}\left(g u_{g} \boldsymbol{f}_{1}\right)+C\left(f_{0}\right)=0, \quad \boldsymbol{f}_{1}=-u_{g} \tau_{1} \nabla_{r} f_{0}$.

$f_{0}(\mathbf{r}, \epsilon)$ and $\boldsymbol{f}_{1}(\mathbf{r}, \epsilon)$ are the $0^{t h}$ and $1^{\text {st }}$-order coefficients of the distribution function, $g(\epsilon)$ is the density of states in silicon, $u_{g}(\epsilon)$ the modulus of the group velocity, $\tau_{1}$ the microscopic relaxation time and $C\left(f_{0}\right)$ the collision term. The main scattering mechanisms in bulk silicon (acoustic and optical phonons, ionized impurities, and impact ionization) are accounted for using the parameter values published in [2]. As for the transport in the inversion layer, the two-dimensional collision mechanisms include surface acoustic phonon scattering, Coulomb scattering with charged centers located near the $\mathrm{Si}_{-} \mathrm{SiO}_{2}$ interface and surface-roughness scattering [4].

\subsection{Modeling of the Gate Current}

When accounting for parallel momentum conservation, the hot-electron current flow from $\mathrm{Si}$ into $\mathrm{SiO}_{2}$ reads [5]

$J_{i n j}(\mathbf{r})=-\frac{1}{2} q \int_{0}^{\frac{\pi}{2}} d \theta \sin \theta \cos \theta \int_{E_{B} / \cos ^{2} \theta}^{\infty} g(\epsilon) u_{g}(\epsilon) f(\mathbf{r}, \epsilon) d \epsilon$

where $\theta$ is the angle between the direction of the group velocity and the unit vector normal to the $\mathrm{Si}_{-} \mathrm{SiO}_{2}$ interface, and $E_{B}$ the barrier height. On the contrary, ignoring 


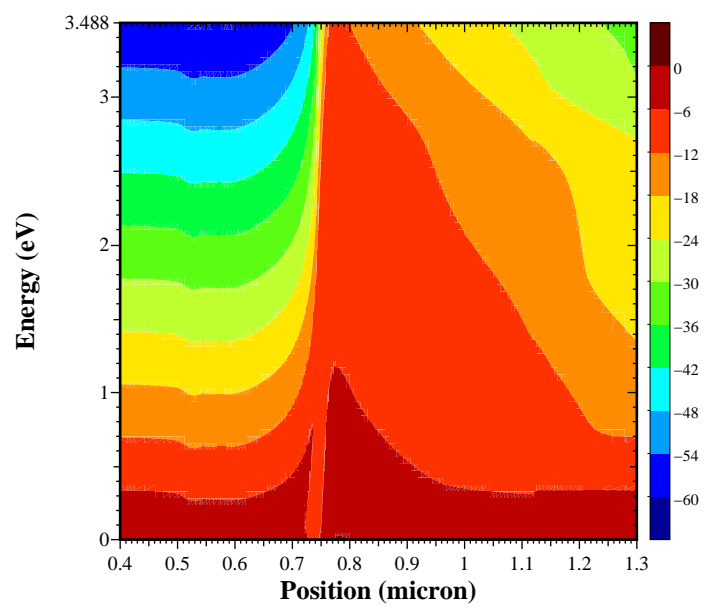

Figure 2: Electron energy distribution along the $\mathrm{Si}_{-} \mathrm{SiO}_{2}$ interface at $V_{c g}=1.0 \mathrm{~V}, V_{f g}=$ $7.0 \mathrm{~V}, V_{d s}=3.3 \mathrm{~V}$.

the conservation of parallel momentum, i.e., allowing all electrons with total energy larger than the barrier height to be injected into $\mathrm{SiO}_{2}$, one finds

$J_{i n j}(\mathbf{r})=-\frac{1}{4} q \int_{E_{B}}^{\infty} g(\epsilon) u_{g}(\epsilon) f(\mathbf{r}, \epsilon) d \epsilon$.

The density of states and the modulus of the group velocity are known from band structure calculations, while the energy distribution function $f=f_{0}[1]$ is provided by the solution of the BTE within the device. A hydrodynamic simulation is carried out to evaluate the energy barrier profile and the current loss factor due to electron scattering in the oxide [5], as well as the potential distribution, which is required as an input for HARM.

\section{Simulation Results and Comparison with Experiments}

Fig. 2 shows the substantial increase of the electron population at high energies under the floating gate in the vicinity of the split-point, thus identifying the location where hot-carrier injection occurs. In Fig. 3a, the floating-gate current has been calculated using Eqs. 2 and 3. Clearly, only when accounting for parallel momentum conservation, the order of magnitude of the current is correctly reproduced. For completeness, the tunneling component, calculated using the WKB method, is shown as well. Its contribution to the total current is found to be negligible with respect to the hot-current one. In Fig. 3b, the floating-gate currents have been evaluated using Eq. 2, without any attempt to adjust the scattering parameters incorporated in HARM. The agreement between simulation and experiment is not perfect, since the calculated currents overestimate the experimental data. However, the dependence of the gate current on the applied voltages is reasonably reproduced. 

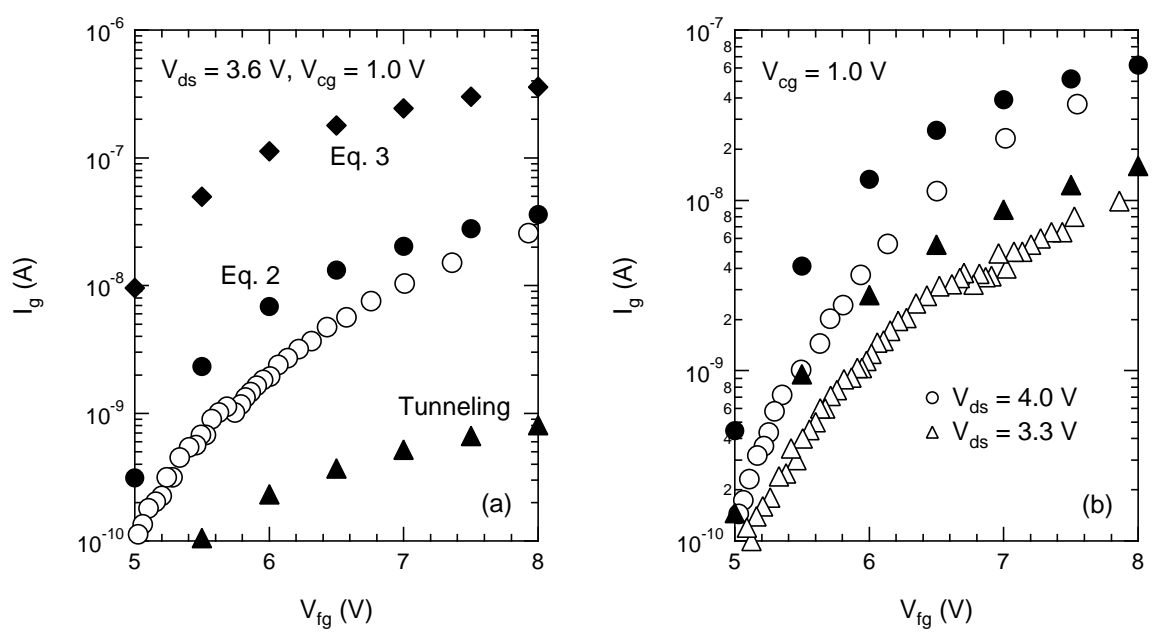

Figure 3: Simulated (filled symbols) and measured (open symbols) floating-gate currents.

\section{Conclusions}

Source-side injection modeling has been tackled starting from the knowledge of the electron energy distribution function at the silicon surface, calculated by the deterministic BTE solver HARM. When accounting for parallel momentum conservation, the calculations reasonably agree with the measurements.

\section{Acknowledgement}

A version of HARM has been provided by Prof. G. Baccarani (University of Bologna, Italy), which the authors gratefully acknowledge.

\section{References}

[1] D. Ventura, A. Gnudi, G. Baccarani, "An efficient method for evaluating the energy distribution of electrons in semiconductors based on spherical harmonics expansion", IEICE Transactions on Electronics, vol. E75-C, pp. 194-199, 1992.

[2] S. Reggiani, M. C. Vecchi, and M. Rudan, "Investigation on Electron and Hole Transport Properties Using the Full-Band Spherical-Harmonics Expansion Method", IEEE Transactions on Electron Devices, vol. 45, pp.2010-2017, 1998.

[3] J. Van Houdt, L. Haspeslagh, D. Wellekens, L. Deferm, G. Groeseneken, and H. E. Maes, "HIMOS-A High Efficiency Flash E ${ }^{2}$ PROM Cell for Embedded Memory Applications", IEEE Transactions on Electron Devices, vol. 40, pp.2255-2263, 1993.

[4] A. Greiner, M. C. Vecchi and M. Rudan, "Modelling surface-scattering effects in the solution of the Boltzmann transport equation based on the spherical-harmonics expansion", Semiconductor Science and Technology, vol. 13, pp. 1080-1089, 1998.

[5] M. V. Fischetti, S. E. Laux, and E. Crabbé, "Understanding hot-electron transport in silicon devices: Is there a shortcut?”, Journal of Applied Physics, vol. 78, pp. 1058-1087, 1995. 